\title{
Feinstaub erhöht Frühgeburtsrisiko
}

\begin{abstract}
Luftverschmutzungen, vor allem durch Feinstaub, können in der Schwangerschaft gefährlich werden. Die Gefahr einer Frühgeburt steigt mit zunehmender Belastung rapide. Dass es einen Zusammenhang zwischen Luftverschmutzung und geringem Geburtsgewicht sowie Frühgeburten gibt, ist schon länger bekannt. Jetzt ist festgestellt worden, welche Schadstoffe beteiligt sind.
\end{abstract}

- Wissenschaftler der University of California haben über 22 Monate lang Schadstoffwerte von sieben staatlichen Messstellen in Los Angeles County ausgewertet. Die Stationen registrierten unter anderem Stickoxide, Ozon, Feinstaub und Kohlenmonoxid. Die Wissenschaftler nutzten zudem Informationen über giftige Chemikalien, die sie von der Luftgüteüberwachung des South Coastal Air Quality Management erhielten. Informationen über Geburten und den
Status der Säuglinge bezogen die Wissenschaftler von der kalifornischen Gesundheitsbehörde. Aus rund 276000 Geburten wurden dabei die 110429 Frauen berücksichtigt, die im Umkreis von fünf Kilometern um eine Luftgütemessstelle lebten.

Gefährlich sind vor allem die polyzyklischen aromatischen Kohlenwasserstoffe (PAK), die im Feinstaub enthalten sind. Sie lassen die Rate der Frühgeburten um bis zu 30\% ansteigen. Hohe Konzentrationen weiterer giftiger Substanzen aus Autoabgasen wie Benzol und Dieselruß erhöhen die Frühgeburtsrate um zusätzlich $10 \%$.

Neben PAK, Benzol und anderen organischen Komponenten habe sich aber auch eine starke Wirkung von Ammoniumnitrat gezeigt Diese Substanz entsteht in der Luft durch Reaktion mehrerer Luftschadstoffe miteinander. Lagen die Konzentrationen dieses Stoffs hoch, stieg der Anteil der Frühgeburten um
21\%. Die Erhöhung des Frühgeburtsrisikos durch Ammoniumnitrat deutet darauf hin, dass auch sekundär entstehende Schadstoffe die Gesundheit der ungeborenen Kinder negativ beeinflussen

\section{Kommentar}

Die Studie habe erstmals auch detailliert die räumlichen und zeitlichen Zusammenhänge von Schadstoffen und Frühgeburten gezeigt. Dies sei eine wichtige Voraussetzung, um die Auswirkungen der Luftverschmutzung auf die öffentliche Gesundheit reduzieren zu können, so die Autoren.

K. MALBeRG =

\footnotetext{
- M. Wilhelm et al.

(Dept of Epidemiology, School of Pubic Health, Univ of California, Los Angeles, USA; E-mail: mwilhelm@ucla.edu)Traffic-related air toxics and preterm birth: a population-based casecontrol study in Los Angeles County, California. Published online on 7 Oct 2011 in Environmental Health $2011 ; 10: 89$ doi:10.1186/1476069X-10-89
}

\section{Vorsicht mit „Ekzemen“ an der Brustwarze}

\section{Eine 91-jährige Frau mit bekannter Familienanamnese für ein Mamma- karzinom wurde wegen eines seit einem Jahr bestehenden Erythems der linken Brust und der Brustwarze in eine senologische Einrichtung überwiesen. Die Überweisungsdia- gnose lautete Morbus Paget oder Ekzem der Brustwarze.}

_ Ein Versuch mit einer steroidhaltigen Salbe war ohne Erfolg geblieben. Bei der Untersuchung zeigte sich eine rötliche, verkrustete Läsion mit partieller Zerstörung der Brustwarze. Die Hautbiopsie bestätigte den Verdacht auf ein lokales Carcinoma in situ und einem Morbus Paget der Brustwarze. Die Patienten unterzog sich einer linksseitigen Mastektomie.

Der Verdacht auf einen Morbus Paget der Brustwarze ist gegeben bei Patien-

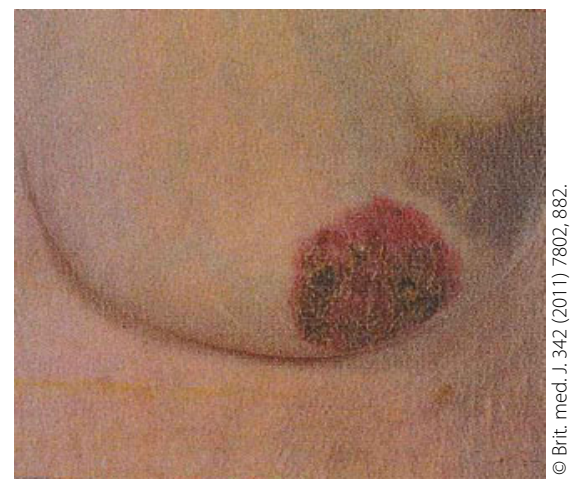

Das „Ekzem“ war ein Karzinom.

tinnen über 50, Einbeziehung des Warzenhofes und der Brustwarze mit Zerstörung der Warzen und keiner Besserung nach einem vierwöchigen Behandlungsversuch mit kortikoidhaltigen Lokalpräparaten. Unter diesen Umständen empfiehlt sich in jedem Fall die Überweisung an ein Brustzentrum.

\section{Kommentar}

Die lange Latenz ist vor allem bei Patientinnen im hohen Lebensalter nicht ungewöhnlich. Viele betagte Frauen haben immer noch eine gewisse Scham, Brüste und Genitale einem Arzt zu präsentieren, ja sie vermeiden es sogar, sich selbst an diesen Körperstellen zu betrachten. Manche sind auch der Ansicht, Gynäkologen wären nur für jüngere Frauen da.

H. S. FÜESSL=

\section{- A. T. W. Ng, T. Irvine}

(Department of Breast Surgery, Royal Surrey County Hospital, Guildford GU2 7XX) Brit. med. J. 342 (2011) 7802, 882. 